\title{
Moments of generalized parton distributions and quark angular momentum of the nucleon
}

\author{
Dirk Brömmel $^{a b}$, Meinulf Göckeler ${ }^{b}$, Philipp Hägler ${ }^{c}$, Roger Horsley ${ }^{d}$, \\ Yoshifumi Nakamura $^{e}$, Munehisa Ohtani ${ }^{*}$, Dirk Pleiter $^{e}$, Paul E.L. Rakow ${ }^{f}$, \\ Andreas Schäfer ${ }^{b}$, Gerrit Schierholz ${ }^{a e}$, Wolfram Schroers ${ }^{e}$, Hinnerk Stüben ${ }^{g}$, and \\ James M. Zanotti ${ }^{d}$ \\ a Deutsches Elektronen-Synchrotoron DESY, 22603 Hamburg, Germany \\ ${ }^{b}$ Institut für Theoretische Physik, Universität Regensburg, 93040 Regensburg, Germany \\ ${ }^{c}$ Institut für Theoretische Physik T39, Physik-Department der TU München, \\ James-Franck-Straße, 85747 Garching, Germany \\ ${ }^{d}$ School of Physics, University of Edinburgh, Edinburgh EH9 3JZ, UK \\ e John von Neuman-Institut für Computing NIC/DESY, 15738 Zeuthen, Germany \\ ${ }^{f}$ Theoretical Physics Division, Department of Mathematical Sciences, University of Liverpool, \\ Liverpool L69 3BX,UK \\ ${ }^{g}$ Konrad-Zuse-Zentrum für Informationstechnik Berlin, 14195 Berlin, Germany \\ E-mail: munehisa.ohtani@physik.uni-regensburg.de
}

\section{QCDSF-UKQCD Collaboration}

\begin{abstract}
The internal structure of hadrons is important for a variety of topics, including the hadron form factors, proton spin and spin asymmetry in polarized proton scattering. For a systematic study generalized parton distributions (GPDs) encode important information on hadron structure in the entire impact parameter space. We report on a computation of nucleon GPDs based on simulations with two dynamical non-perturbatively improved Wilson quarks with pion masses down to $350 \mathrm{MeV}$. We present results for the total angular momentum of quarks with chiral extrapolation based on covariant baryon chiral perturbation theory.
\end{abstract}

The XXV International Symposium on Lattice Field Theory

July 30 - August 42007

Regensburg, Germany

\footnotetext{
${ }^{*}$ Speaker.
} 


\section{Introduction}

The internal structure of nucleons has attracted much attention in the contexts of the nucleon form factors, proton spin and spin/charge asymmetry in deeply virtual Compton scattering and so on. For a systematic study of the nucleon internal structure, generalized parton distributions (GPDs) are introduced through the off-forward matrix elements of quark-bilinear operators:

$$
\int \frac{d \eta}{4 \pi} e^{i \eta x}\left\langle P^{\prime}\left|\bar{q}\left(-\frac{\eta n}{2}\right) \gamma^{\mu} \mathscr{U} q\left(\frac{\eta n}{2}\right)\right| P\right\rangle=\bar{N}\left(P^{\prime}\right)\left(\gamma^{\mu} H(x, \xi, t)+i \frac{\sigma^{\mu v} \Delta_{v}}{2 M} E(x, \xi, t)\right) N(P),
$$

with a light cone vector $n$ and the momentum transfer $\Delta=P^{\prime}-P$ as functions of the quark momentum fraction $x$, the skewedness $\xi=-n \cdot \Delta / 2$ and the virtuality $t=\Delta^{2}$. The axial counterparts are denoted by $\tilde{H}$ and $\tilde{E}$. Since the GPD is defined with the finite momentum transfer in contrast to the conventional parton distribution functions, partons bring us the informations on hadron structure in the transverse space.

In this contribution, we report on the first moments of GPD, so called generalized form factors, for nucleon, as a function of the virtuality calculated on the lattice with unquenched configurations of QCDSF/UKQCD collaboration.

In the forward limit these generalized form factors provide the total angular momentum of quark in the nucleon through Ji's sum rule [1],

$$
J^{q}=\frac{1}{2} \int_{-1}^{1} d x x(H(x, \xi, 0)+E(x, \xi, 0)) \equiv \frac{1}{2}\left(A_{20}(t=0)+B_{20}(t=0)\right) .
$$

Combined with the quark spin contributions to the nucleon obtained as the forward value of the axial form factor,

$$
s^{q}=\frac{1}{2} \int_{-1}^{1} d x \tilde{H}(x, \xi, 0) \equiv \frac{1}{2} \tilde{A}_{10}(t=0),
$$

we compute the orbital angular momentum of quarks as $L^{q}=J^{q}-s^{q}$. Using the results of chiral perturbation theory $(\chi \mathrm{PT})$ for chiral extrapolation to the physical point, we discuss the angular momentum carried by quark in the nucleon.

\section{Generalized form factors on the lattice}

The Mellin moments of the GPDs are known to be expressed by polynomials in terms of $\xi$ [2],

$$
\int_{-1}^{1} d x x^{n-1}\left[\begin{array}{c}
H(x, \xi, t) \\
E(x, \xi, t)
\end{array}\right]=\sum_{k=0}^{[(n-1) / 2]}(2 \xi)^{2 k}\left[\begin{array}{l}
A_{n, 2 k}(t) \\
B_{n, 2 k}(t)
\end{array}\right] \pm \delta_{n, \text { even }}(2 \xi)^{n} C_{n}(t) .
$$

The generalized form factors $A_{n, 2 k}, B_{n, 2 k}$ and $C_{n}$ are defined from the coefficients of this expansion. Since the integration by $x$ makes the quark operator local, the $(n-1)$-th moments can be calculated [3] on the lattice through the matrix element of $\left\langle P^{\prime}\left|\bar{q} \gamma^{\left\{\mu_{1}\right.} D^{\mu_{2}} \ldots D^{\left.\mu_{n}\right\}} q\right| P\right\rangle$ by taking a ratio of the three- and two-point functions.

To estimate these correlation functions, 400 to 2200 configurations are used for each $\beta, \kappa$ with two flavor Wilson fermion with the clover improvement. Simulations are performed with various set of parameters $\beta$ and $\kappa$ corresponding to the lattice spacing less than $0.09 \mathrm{fm}$ and pion mass covering from order of $1 \mathrm{GeV}$ down to $350 \mathrm{MeV}$ with a reference scale $r_{0}=0.467 \mathrm{fm}$. Nonperturbative 

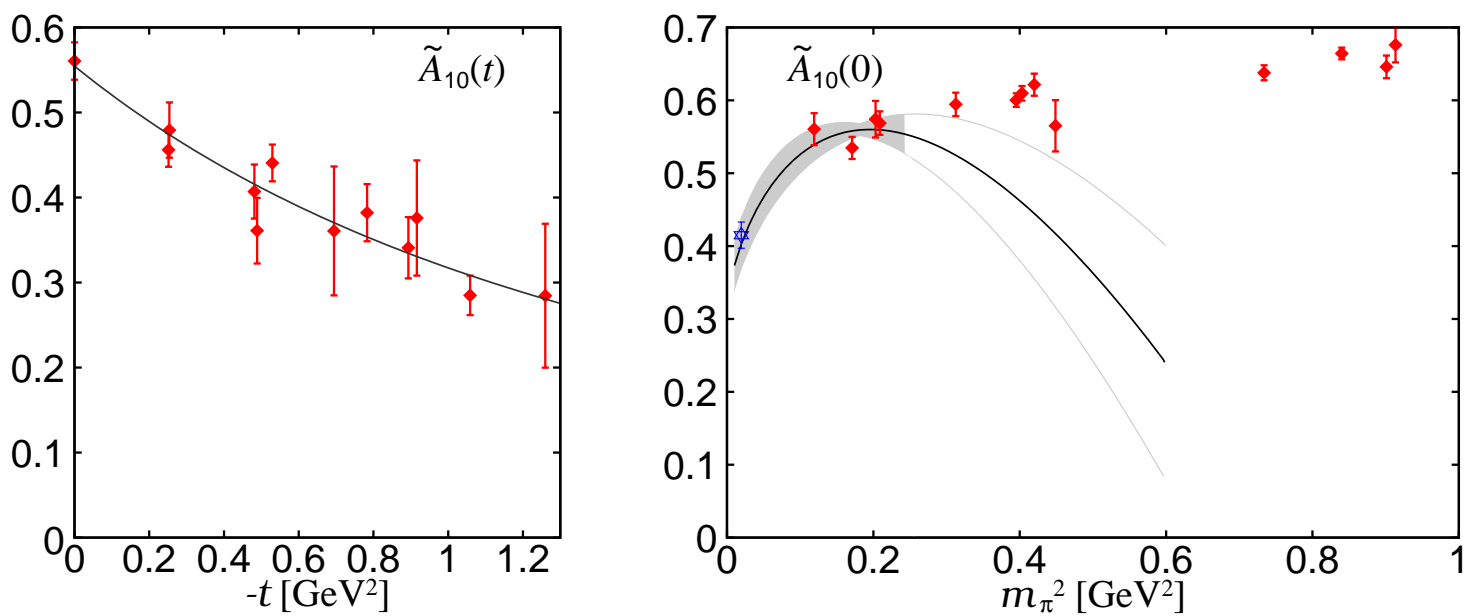

Figure 1: The isoscalar axial form factor $\tilde{A}_{10}(t)$ for $\beta=5.29, \kappa=0.13632$ with dipole fit (left) and the forward values with $\chi \mathrm{PT}$ fit (right). The open star in the right panel represents the latest experimental value of HERMES.

renormalizations are incorporated to convert the lattice results into the values in the $\overline{\mathrm{MS}}$ scheme at a scale of $\mu^{2}=4 \mathrm{GeV}^{2}$. The $\mathscr{O}(a)$ improvement of the quark energy-momentum tensor are carried out through the boosted perturbation theory following ref.[4] and the tadpole improved version is used for the axial current following ref.[5]. We note that the contributions from disconnected diagrams are not included in the present lattice results.

\section{Lattice simulation results and chiral extrapolation}

We focus on the generalized form factors $A_{20}$ and $B_{20}$ as well as the axial form factor $\tilde{A}_{10}$ to evaluate the quark angular momentum in the nucleon.

Typical $t$ dependence of the axial form factor in the isoscalar channel are shown in Fig 1 . The obtained lattice data agrees well with a fitting by the dipole form, $\tilde{A}_{10}(0) /\left(1-t / \mathrm{m}^{2}\right)^{2}$. The forward values obtained by setting $t=0$ present a smooth pion-mass dependence as shown in the right panel of Fig.1.

Here we use an expression derived in a heavy baryon $\chi \mathrm{PT}[6]$,

$$
\tilde{A}_{n, k}^{\mathrm{u}+\mathrm{d}}(0)=\alpha_{n, k}\left[1-\frac{3 m_{\pi}^{2} g_{A}^{2}}{16 \pi^{2} F_{\pi}^{2}}\left(\ln \frac{m_{\pi}^{2}}{\lambda^{2}}+1\right)\right]+\beta_{n, k} m_{\pi}^{2}+\mathscr{O}\left(m_{\pi}^{3}\right),
$$

for the chiral extrapolation with fitting parameters $\alpha_{10}$ and $\beta_{10}$ at a scale of $\lambda=1 \mathrm{GeV}$. As the heavy baryon formalism is valid only for the small pion mass, we restrict the data points at pion masses less than $500 \mathrm{MeV}$. Then it turns out that the chiral $\log$ term gives a strong $m_{\pi}$ dependence for small $m_{\pi}$ region and the extrapolated value is eventually comparable with the latest experimental value of deep-inelastic scattering reported by HERMES [7]. With this extrapolation, we obtain the quark spin contribution in the nucleon as $\tilde{A}_{10}^{\mathrm{u}+\mathrm{d}}(0) \equiv 2 s^{\mathrm{u}+\mathrm{d}}=0.402 \pm 0.024$ at the physical pion mass.

Next we show a typical $t$ dependence of generalized form factors $A_{20}, B_{20}$ and $C_{2}$ in the isovector channel in Fig.2, Up to $1 \mathrm{GeV}^{2}$, the lattice results of $A_{20}$ agree with the dipole fit $A_{20}(0) /\left(1-t / m_{\mathrm{D}}^{2}\right)^{2}$. The dipole mass $m_{\mathrm{D}}$ of $A_{20}$ shows a smooth $m_{\pi}$ dependence shown in the 

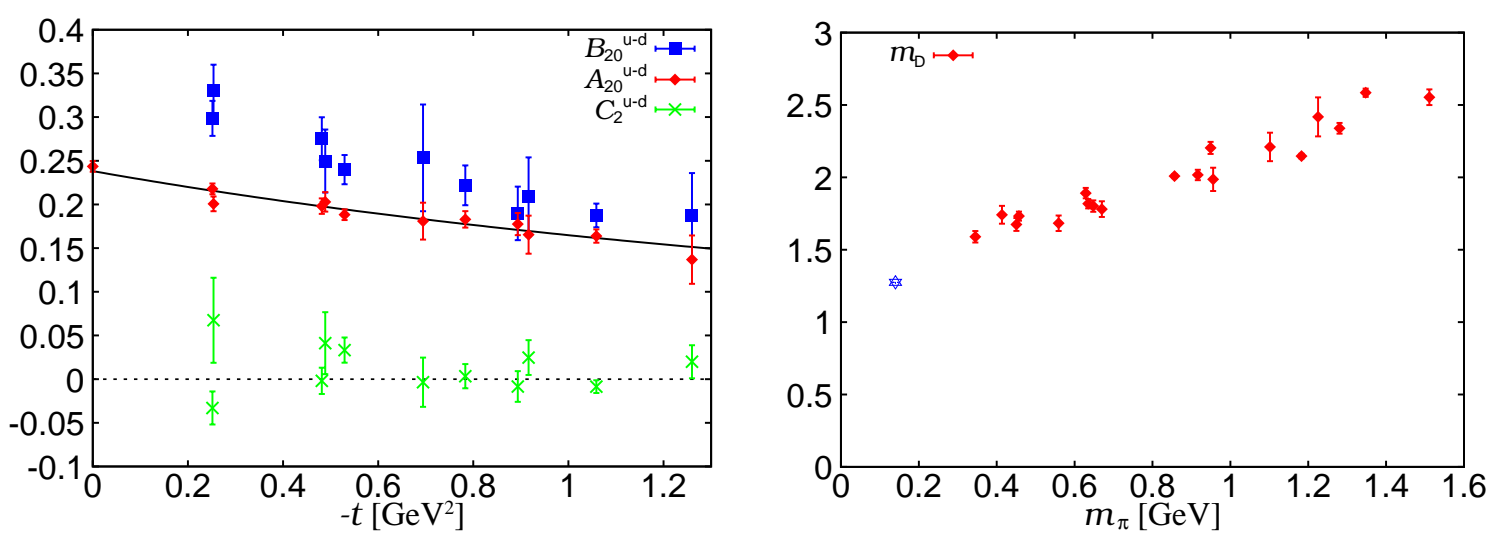

Figure 2: Generalized form factors in the isovector channel for $\beta=5.29, \kappa=0.13632$ with dipole fit for $A_{20}$ and the dipole mass of $A_{20}$. The open star represents the experimental value of $f_{2}$ tensor meson mass.

right panel of Fig.2. We see that the dipole mass seems to extrapolate to the observed mass of tensor meson $f_{2}$ at the physical point. This fact contrasts with a mass scale of the electromagnetic form factors comparable with the vector meson mass [8].

However, the empirical dipole fit for the generalized form factors has no solid justification from a theoretical point of view. Therefore we count on the covariant baryon chiral perturbation [9] to extract the forward values of $B_{20}$ as well as the chiral extrapolation of $A_{20}$ and $B_{20}$.

The forward values of $A_{20}$ are identical to the quark momentum fraction $\langle x\rangle^{q}$ and are shown as a function of $m_{\pi}^{2}$ in Fig 3 . Both in the isoscalar and isovector channel, the lattice results show a moderate pion mass dependence. These values are extrapolated to the physical point by the following eqs. derived in the baryon $\chi \mathrm{PT}$,

$$
\begin{aligned}
A_{2,0}^{\mathrm{u}+\mathrm{d}}(0)= & a_{20}^{\mathrm{s}}+c_{9} \frac{4 m_{\pi}^{2}}{M_{0}^{2}}-a_{20}^{\mathrm{s}} \frac{3 g_{A}^{2} m_{\pi}^{2}}{16 \pi^{2} F_{\pi}^{2}}\left[\frac{m_{\pi}^{2}}{M_{0}^{2}}+\frac{m_{\pi}^{2}}{M_{0}^{2}}\left(2-\frac{m_{\pi}^{2}}{M_{0}^{2}}\right) \ln \frac{m_{\pi}}{M_{0}}\right. \\
& \left.+\frac{m_{\pi}}{\sqrt{4 M_{0}^{2}-m_{\pi}^{2}}}\left(2-4 \frac{m_{\pi}^{2}}{M_{0}^{2}}+\frac{m_{\pi}^{4}}{M_{0}^{4}}\right) \arccos \frac{m_{\pi}}{2 M_{0}}\right]+\mathscr{O}\left(p^{3}\right)
\end{aligned}
$$
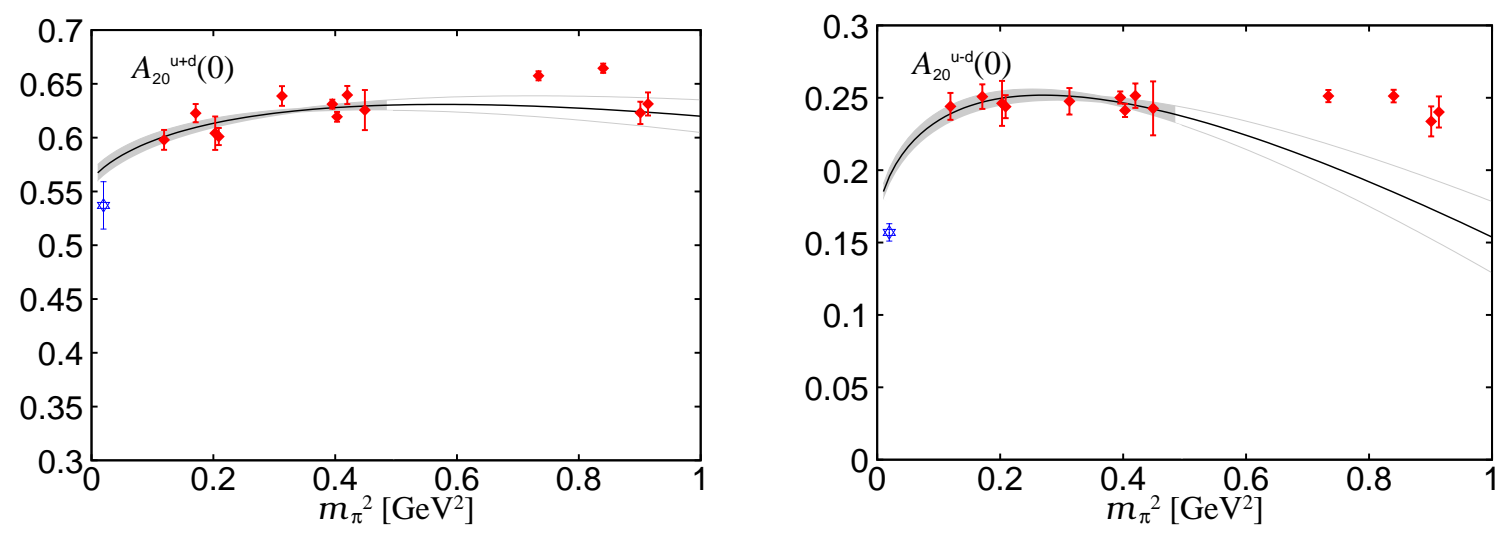

Figure 3: The forward values of $A_{20}$ in the isoscalar (left) and isovector (right) channel with $\chi$ PT fits. The open stars represent the phenomenological values from CTEQ6. 

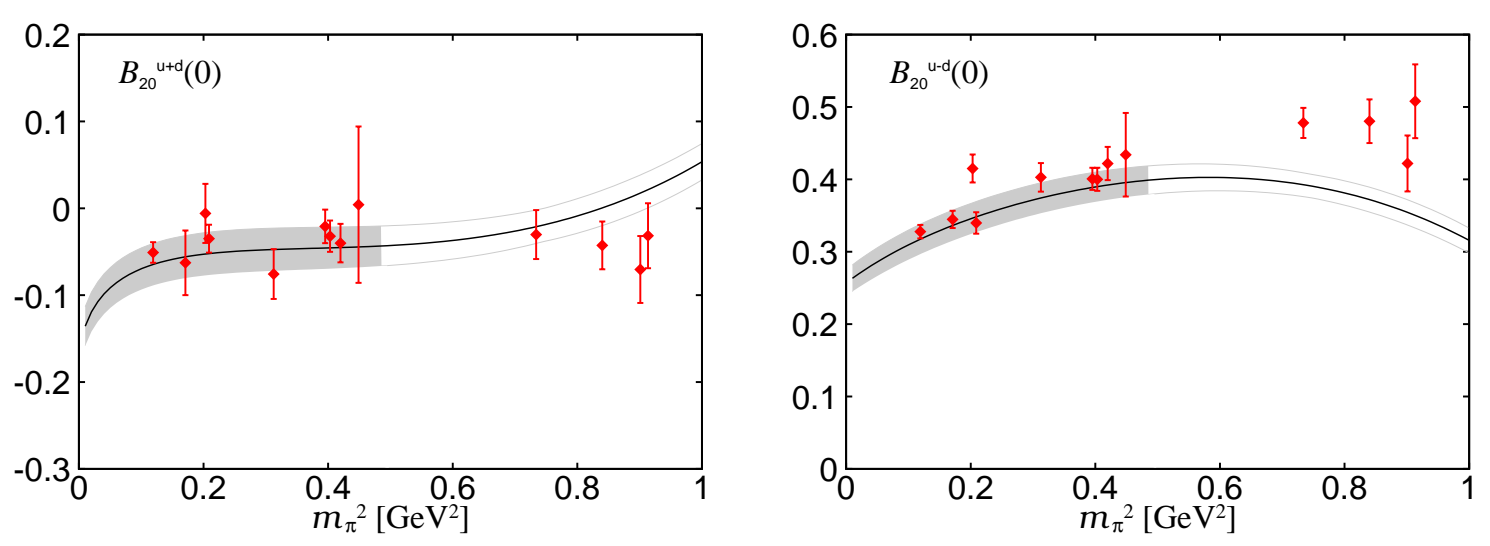

Figure 4: The forward values of $B_{20}$ extrapolated by $\chi \mathrm{PT}$ in the isoscalar (left) and isovector (right) channel with $\chi$ PT fits.

for the isoscalar channel and

$$
\begin{aligned}
A_{2,0}^{\mathrm{u}-\mathrm{d}}(0)= & a_{20}^{\mathrm{v}}+c_{8} \frac{4 m_{\pi}^{2}}{M_{0}^{2}}+a_{20}^{\mathrm{v}} \frac{g_{A}^{2} m_{\pi}^{2}}{16 \pi^{2} F_{\pi}^{2}}\left[-\left(3+\frac{1}{g_{A}^{2}}\right) \ln \frac{m_{\pi}^{2}}{\lambda^{2}}+\frac{m_{\pi}^{2}}{M_{0}^{2}}-2+\frac{m_{\pi}^{2}}{M_{0}^{2}}\left(6-\frac{m_{\pi}^{2}}{M_{0}^{2}}\right) \ln \frac{m_{\pi}}{M_{0}}\right. \\
& \left.+\frac{m_{\pi}}{\sqrt{4 M_{0}^{2}-m_{\pi}^{2}}}\left(14-8 \frac{m_{\pi}^{2}}{M_{0}^{2}}+\frac{m_{\pi}^{4}}{M_{0}^{4}}\right) \arccos \frac{m_{\pi}}{2 M_{0}}\right] \\
& +\Delta a_{20}^{\mathrm{v}} \frac{g_{A}^{2} m_{\pi}^{2}}{48 \pi^{2} F_{\pi}^{2}}\left[2 \frac{m_{\pi}^{2}}{M_{0}^{2}}+\frac{m_{\pi}^{2}}{M_{0}^{2}}\left(6-\frac{m_{\pi}^{2}}{M_{0}^{2}}\right) \ln \frac{m_{\pi}^{2}}{M_{0}^{2}}+2 m_{\pi} \frac{\left(4 M_{0}^{2}-m_{\pi}^{2}\right)^{3 / 2}}{M_{0}^{4}} \arccos \frac{m_{\pi}}{2 M_{0}}\right]
\end{aligned}
$$

for the isovector channel, where $M_{0}$ is the nucleon mass in the chiral limit. We perform 2-parameter fits with $a_{20}^{\mathrm{s}}, c_{9}$ for the isoscalar and $a_{20}^{\mathrm{v}}, c_{8}$ for the isovector channel at a scale of $\lambda=1 \mathrm{GeV}$ and fixed the other values following ref.[9]. A strong $m_{\pi}$ dependence is observed especially for the isovector channel, but the extrapolated values in both channels overshoot beyond the phenomenological values estimated using the CTEQ6 parton distribution functions. The chiral extrapolation gives $A_{20}^{\mathrm{u}+\mathrm{d}} \equiv\langle x\rangle^{\mathrm{u}+\mathrm{d}}=0.572 \pm 0.012$ for the isoscalar channel and $A_{20}^{\mathrm{u}-\mathrm{d}} \equiv\langle x\rangle^{\mathrm{u}-\mathrm{d}}=0.198 \pm 0.008$ for the isovector channel at the physical pion mass. See ref.[10] for discretization effects of these form factors.

In contrast to $A_{20}$, the forward values of $B_{20}$ cannot be calculated directly from the lattice simulation since the kinematic pre-factor for $B_{20}$ vanishes at zero momentum transfer. Again we use the expressions of covariant baryon $\chi \mathrm{PT}[9]$,

$$
\begin{aligned}
& B_{2,0}^{\mathrm{u} \pm \mathrm{d}}(t)=\left(b_{20}^{\mathrm{s}, \mathrm{v}}+\hat{\delta}_{B}^{\mathrm{s}, \mathrm{v}} m_{\pi}^{2}+\hat{\delta}_{B t}^{\mathrm{s}, \mathrm{v}} t\right) \frac{M_{\mathrm{N}}\left(m_{\pi}\right)}{M_{0}} \mp a_{20}^{\mathrm{s}, \mathrm{v}} \frac{(2 \pm 1) g_{A}^{2} M_{0}^{2}}{48 \pi^{2} F_{\pi}^{2}} G(t), \\
& G(t)=\int_{-\frac{1}{2}}^{\frac{1}{2}} \frac{d u}{\tilde{M}^{8}}\left[\left(M_{0}^{2}-\tilde{M}^{2}\right) \tilde{M}^{6}+9 m_{\pi}^{2} M_{0}^{2} \tilde{M}^{4}-6 m_{\pi}^{4} M_{0}^{2} \tilde{M}^{2}+6 m_{\pi}^{2} M_{0}^{2}\left(m_{\pi}^{4}-3 m_{\pi}^{2} \tilde{M}^{2}+\tilde{M}^{4}\right) \ln \frac{m_{\pi}}{\tilde{M}}\right. \\
& \left.-\frac{6 m_{\pi}^{3} M_{0}^{2}}{\sqrt{4 \tilde{M}^{2}-m_{\pi}^{2}}}\left(m_{\pi}^{4}-5 m_{\pi}^{2} \tilde{M}^{2}+5 \tilde{M}^{4}\right) \arccos \frac{m_{\pi}}{2 \tilde{M}}\right]\left.\right|_{\tilde{M}^{2}=M_{0}^{2}+\left(u^{2}-\frac{1}{4}\right) t},
\end{aligned}
$$

to fit the lattice results as a function of $t$ and $m_{\pi}$. Free fit parameters are $b_{20}^{\mathrm{s}, \mathrm{v}}, \hat{\delta}_{B}^{\mathrm{s}, \mathrm{v}}$ and $\hat{\delta}_{B t}^{\mathrm{s}, \mathrm{v}}$ for the isoscalar and isovector channel respectively, and the parameters of $a_{20}^{\mathrm{s}, \mathrm{v}}$ are fixed by the fitting of 

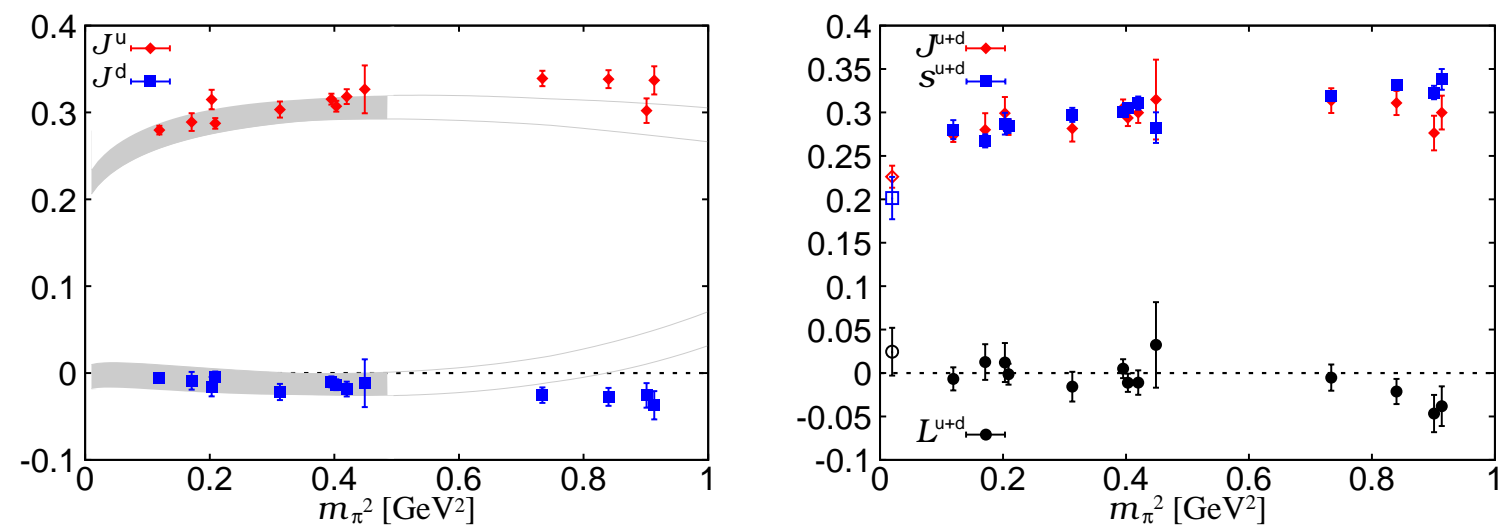

Figure 5: Total angular momentum of quark in the nucleon with $\chi \mathrm{PT}$ fit (left) and spin, orbital angular momentum of quarks (right). The open symbols represent the extrapolated values to the physical pion mass.

$A_{20}^{\mathrm{u} \pm \mathrm{d}}$. The forward values of $B_{20}$ extracted from this fit with fixed $m_{\pi}$ are shown in Fig 4 , where the solid lines represent the section of fitting surfaces at $t=0$.

Since the forward value of $B_{20}^{\mathrm{u}+\mathrm{d}}$ is equivalent to the difference of $2 J^{q}-\langle x\rangle^{q}$, the small values of the lattice results indicate the cancellation between total angular momentum and momentum fraction of quarks. However, the $\chi$ PT fit suggests a sizeable bending through the chiral extrapolation, which makes a sharp contrast to the chiral quark soliton model [11]. We obtain $B_{20}^{\mathrm{u}+\mathrm{d}}(0)=$ $-0.120 \pm 0.023$ at the physical point for the isoscalar channel and $B_{20}^{\mathrm{u}-\mathrm{d}}(0)=0.269 \pm 0.020$ for the isovector channel.

Combining all these data, we can estimate the angular momentum of quarks in the nucleon. The pion mass dependence of the total angular momentum $J^{q}$ for $\mathrm{u}$ and d quark is shown in Fig.5. The strong $m_{\pi}$ dependences of $A_{20}$ in the isovector channel and $B_{20}$ in the isoscalar channel are enhanced for the $\mathrm{u}$ quark and gives a significant suppression of $J^{\mathrm{u}}$ near the physical point, while these dependences cancel each other for the d quark and so keep the value of $J^{\mathrm{d}}$ small. The extrapolation to the physical point gives $J^{\mathrm{u}}=0.230 \pm 0.008$ and $J^{\mathrm{d}}=-0.004 \pm 0.008$.

The quark spin $s^{q}$, the total and orbital angular momentum $L^{q}=J^{q}-s^{q}$ are shown in the right panel of Fig.5. We obtain the values at physical point by the chiral extrapolation as $J^{\mathrm{u}+\mathrm{d}}=0.226 \pm$ $0.013, s^{\mathrm{u}+\mathrm{d}}=0.201 \pm 0.024$ and $L^{\mathrm{u}+\mathrm{d}}=0.025 \pm 0.027$. The results show that the total angular momentum of quark is comparable with the quark spin and hence the orbital angular momentum is consistent with zero, which agrees with the result of ref.[12].

\section{Conclusions}

We have carried out lattice simulations to calculate the first moments of GPDs, which play an important role for the proton spin, quark transverse density and deeply virtual Compton scattering. The lattice results of the generalized form factor $A_{20}$ are fitted to the dipole form for small $-t$ region and the dipole mass turns out to be comparable with the observed tensor meson mass. Since this empirical fit has no solid justification from a theoretical point of view, we employ baryon $\chi \mathrm{PT}$ to take the forward limit of $B_{20}$ and to chirally extrapolate the form factors to the physical pion mass. The resulting values indicate that the total angular momentum of quarks in the nucleon is of the 
same size as the quark spin contribution, while the orbital angular momentum is consistent with zero.

Further analyses are needed to estimate the finite size effects [13], contributions from disconnected diagrams and so on. Results with lighter pion masses will be forthcoming.

\section{Acknowledgments}

The numerical calculations have been performed on the Hitachi SR8000 at LRZ (Munich), the BlueGene/L and the Cray T3E at EPCC (Edinburgh), the BlueGene/Ls at NIC/FZJ (Jülich) and KEK (by the Kanazawa group as part of DIK research program) and on the APEmille and apeNEXT at NIC/DESY (Zeuthen). This work was supported in part by the DFG and by the EU Integrated Infrastructure Initiative Hadron Physics (I3HP) under contract number RII3-CT-2004-506078.

\section{References}

[1] X.-D. Ji, Gauge-Invariant Decomposition of Nucleon Spin, Phys. Rev. Lett. 78 (1997) 610 [hep-ph/9603249].

[2] X.-D. Ji, Off forward parton distributions, J. Phys. G24 (1998) 1181 [hep-ph/9807358].

[3] M. Göckeler et al. [QCDSF collaboration], Generalized Parton Distributions from Lattice QCD, Phys. Rev. Lett. 92 (2004) 042002 [hep-ph / 030424 9];

$\mathrm{Ph}$. Hägler et al. [LHPC collaboration], Moments of nucleon generalized parton distributions in lattice QCD, Phys. Rev. D68 (2003) 034505 [hep-lat/ 0304018 ].

[4] S. Capitani et al., Renormalisation and off-shell improvement in lattice perturbation theory, Nucl. Phys. B 593 (2001) 183 [hep-lat/ 000700 4].

[5] A. Ali Khan et al. [QCDSF collaboration], Axial coupling constant of the nucleon for two flavors of dynamical quarks in finite and infinite volume, Phys. Rev. D74 (2006) 094508 [hep-lat/0603028].

[6] M. Diehl, A. Manashov, and A. Schäfer, Chiral perturbation theory for nucleon generalized parton distributions, Euro. Phys. J. A29 (2006) 315 [hep-ph/0608113].

[7] A. Airapetian et al. [HERMES Collaboration], Precise determination of the spin structure function $g_{1}$ of the proton, deuteron, and neutron, Phys. Rev. D75 (2007) 012007.

[8] W. Schroers et al. [QCDSF/UKQCD Collaboration], Nucleon electromagnetic form factors with Wilson fermions, POS (LATTICE 2007) 161.

[9] M. Dorati, T. A. Gail and T. R. Hemmert, Chiral perturbation theory and the first moments of the generalized parton distributions in a nucleon, nucl-th/0703073.

[10] D. Pleiter et al. [QCDSF/UKQCD Collaboration], Probing the chiral limit with clover fermions II: The baryon sector, POS (LATTICE 2007) 129.

[11] K. Goeke et al., The pion mass dependence of the nucleon form-factors of the energy momentum tensor in the chiral quark-soliton model, Phys. Rev. C75 (2007) 055207 [hep-ph/ 0702031$].$

[12] Ph. Hägler et al. [LHPC Collaboration], Nucleon Generalized Parton Distributions from Full Lattice $Q C D$, arXiv:0705.4295[hep-lat].

[13] W. Detmold and C.-J. David Lin, Twist-two matrix elements at finite and infinite volume, Phys. Rev. D71 (2005) 054510 [hep-lat/ 0501007$].$ 